$\xi=-1$

\title{
Priority based Dynamic Resource Allocation and Scheduling for Wimax Networks
}

\author{
${ }^{1}$ Ms. P. Sudha, ${ }^{2}$ Dr. A. Rengarajan \\ ${ }^{1}$ Research scholar, Bharathiar University, Coimbatore, Tamil Nadu, India \\ Mobile: 9486279814 \\ ${ }^{2}$ Professor, Department of CSE, Vel Tech Multitech Dr. RS Engineering College, Avadi, Chennai, \\ Tamil Nadu, India \\ Mobile: 8056133788 , \\ *Corresponding author E-mail: ${ }^{1}$ kannansudha2001@gmail.com, ${ }^{2}$ rengu_rajan@yahoo
}

\begin{abstract}
WiMAX network provides effective internet communication between users with low expense and ease of deployment. It is used efficiently in small to medium enterprises. However, proficient resource allocation and scheduling is still a critical requirement in WiMAX networks due to the varying factors related to the network communication. If the network resources are not appropriately allocated, then there are possibilities for missing out critical data, or wasting more resources on transmitting less important data which in turn will have adverse affect on data transmission in the future stages. Hence, in this paper, we propose to develop a Priority Based Dynamic Resource Allocation and Scheduling for WiMAX Networks. In this technique, the incoming data are dynamically allocated, and then users are prioritized to identify the critical users. The allocation and scheduling is performed by considering user priority, robustness and also power consumption rate to ensure effective network performance.
\end{abstract}

Keywords:

\section{Introduction}

\subsection{WiMAX Networks}

Worldwide Interoperability for Microwave Access (WiMAX) is also known as the IEEE 802.16 standard. WiMAX enables the access to metropolitan area networks (WMAN) wirelessly in an inexpensive manner [1]. WiMAX is referred as last mile solution since it facilitates quick connectivity to the network. Also, WiMAX provides cables and fibers with better capacity inexpensively [2]. Orthogonal Frequency Division Multiple Access (OFDMA) is the technique used in the working of WiMAX. WiMAX operates at a bandwidth between $1.25 \mathrm{MHz}$ to $28 \mathrm{MHz}$ of the frequency spectrum. Enhanced antenna mechanisms such as multiple input multiple output (MIMO) is also used by the WiMAX network.

Time division multiplexing and frequency division multiplexing techniques such as TDD and FDD are used in the WiMAX networking. Effective coding protocols such as Space-time coding and turbo coding are also incorporated in the WiMAX technology to enhance the security options. Several QoS features are supported by the WiMAX technology which facilitates voice, data as well as video services [3]. Upto medium level enterprise subscribers, WiMAX is capable of providing internet access at minimal expense, which is aided by applications like VoIP, video conferencing and web [2]. Internet Protocol Television (IPTV) is the most noteworthy application in the WiMAX technology, which is basically a multimedia application; with its QoS requirement being high performance [4].

\subsection{Wimax Classification}

In the WiMAX network, the QoS services are classified into five classes. The classification is performed on the basis of various QoS needs. The five classes are given below [5]:

Unsolicited Grant Service (UGS): The traffic with constant bit rate (CBR) are considered in this class. For instance: Voice applications like UGS services

Real-Time Polling Service (rtPS): The real time traffic with variable size data packets (VBR) at regular time intervals are included in this group. Example: MPEG and video.

Extended Real-Time Polling Service (ertPS): Real time applications with strict constraints like data rate, delay assurance, etc is included in this service class. Example: VoIP along with silent suppression.

Non-Real-Time Polling Service (nrtPS): Applications which involve traffic flow of variable sized packets are included in this service class. This service class also facilitates features like delay tolerance as well as minimum data rate. Example: FTP.

Best Effort (BE): Traffic flows which do not stipulate any QoS requirement are included in this service class. Example: HTTP.

\subsection{Scheduling in WiMAX}

Resource Allocation is a process of dividing the currently accessible resources and allocating it to required users. In the dynamic resource allocation process, the radio resources are allocated on the basis of the existing signal strength of the user. 
Based on the separation between the base station and the endorser, the flag quality of every client is assessed. The allocation of the resources is performed in the real time [6].

Scheduling is a process in which the resources are distributed between existing users in a reasonable way. In scheduling, the aim is to increase the throughput along with minimum delay as well as to ensure fairness between every available user. In scheduling technique, while ensuring fairness, the queue size and the packet priority are the critical factors [4].

The planning procedure is arranged into four classifications. They are unified, disseminated, facilitated appropriated and awkward dispersed booking. In the centralized scheduling technique, the BS makes scheduling decisions which are collision free and then accordingly schedules it. In the distributed scheduling technique, the resources are scheduled in a three way handshake method, which includes request, grant and confirm messages. In the coordinated distributed scheduling technique, the resources are scheduled without consulting the base station and such that to ensure free of contention [7].

In WiMAX network, developing a scheduling algorithm which ensures fairness and carries out all functionalities is a hard task due to several levels of QoS services available [8]. In wireless networks, the resources are usually limited. For example: bandwidth and the transmission rate in the wireless networks. Due to these restraints, developing a scheduling algorithm becomes more complicated [9]. An ideal scheduling algorithm can increase the throughput and reduce the overhead after satisfying the limited bandwidth, delay and jitter constraints of WiMAX network [7] However, it is a hard task to determine the traffic features and service needs of the various applications available in WiMAX that use the resources [10].

\section{Related Works}

Tri M Nguyen et al [11] have proposed QoS-aware dynamic resource allocation for wireless broadband access networks. This paper presents a QDRA scheme which enhances the QoS in all the service types present in the WiMAX network. The QDRA technique will dynamically regulate the DL/UL subframe ratio on the basis of the available DL and UL bandwidth and also consider the priority of the available traffic. This improves the bandwidth utilization in the network. When compared with the conventional priority based techniques, the proposed QDRA scheme shows improvement in the performance of the rtPS, nrtPS, and BE traffic. The QDRA scheme fulfills all the QoS requirements for every service class.

Yao-Hsing Chung et al [12] have proposed a Balanced Resource Scheduling (BRS) Scheme with Adaptive Priority Thresholds for OFDMA Downlink Systems. The BRS conspire has versatile need edge for the OFDMA downlink frameworks. The BRS scheme has adaptive priority threshold for the OFDMA downlink systems. To precisely handle the tradeoff among improving the system throughput as well as QoS requirement assurance, the BRS technique applies a priority threshold to every user in the network. The BRS technique is basically a two phase scheduling process. In the main stage, a need based RA calculation is performed. In the second stage, a CSI based RA calculation is performed.

Hamed Banizaman et al [13] have proposed Dynamic resource allocation algorithm in multi-user cooperative OFDMA systems: considering QoS and fairness constraints. Initially, the dynamic resource allocation issue in the multi-user DF cooperative OFDMA systems is considered. Next, a bit assignment algorithm is demonstrated which ensures fairness in assigning sub channel along with power minimization factor. This algorithm targets to reduce the transmission power by considering the BER as well as the data rate factors. Then a three staged allocation algorithm is presented which ensures fairness with minimum complexity, while the algorithm was developed assuming higher transmission power, higher battery level, data rate and BER.

\section{Priority Based Dynamic Resource Allocation and Scheduling}

\subsection{Overview}

In this paper, we propose to plan need based dynamic asset assignment and planning for WiMAX systems. At first subframes are apportioned in view of Downlink (DL) and Uplink (UL) proportion [11]. The base station (BS) progressively modifies the DL/UL subframe proportion in view of the approaching activity (DL information movement and UL ask). The activity is ordered as Real-time (RT), Non ongoing (NRT) and Best exertion (BE). The QoS prerequisites of RT clients are mostly greatest postpone resilience and greatest reasonable bundle dropping rate. Additionally for NRT clients, the base transmission rate is the QoS prerequisite. Then priorities are assigned to the users according to the type of traffic [12]. The sub channels are then sorted based on the channel state indicator (CSI) values. Then rates are assigned to the users based on their priority and CSI values of the sub channels.

The end goal to limit the transmission control, in every emphasis, the measure of energy lessened for a low need client because of sub-channel portion is ascertained. Transmission power ought to be limited for the client with minimum need. On the off chance that apportioning more sub-channels to a client does not diminish its transmission control, the client is expelled from the distribution procedure [13].

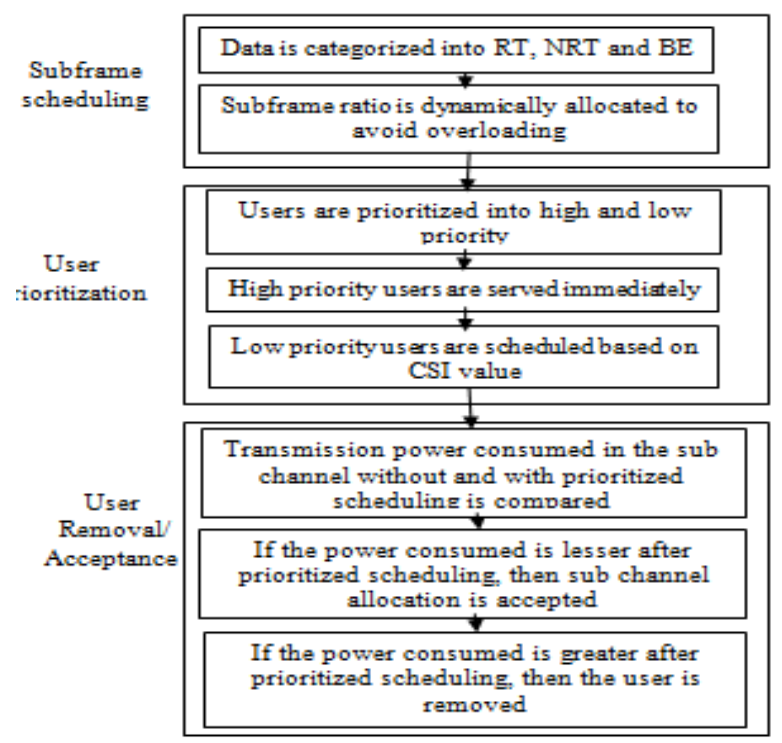

Fig.1: Block Diagram

\subsection{Subframe Scheduling}

For efficient network performance, the appropriate scheduling of the data is critical. The Base Station (BS) initially allocates the subframe ratio based on the incoming DL data traffic as well as the UL request traffic. The subframe scheduling is performed dynamically since the traffic includes different data types such as Real Time, Non Real Time and Best Effort; a fixed subframe DL/UL ratio is not taken as it might overload the channel. This process is described in algorithm 1 .
Algorithm 1
Notations
1. DL : Downlink
2. UL : Upper link
3. BS : Base Station
4. RT : Real Time 
5. NRT: Non Real Time

6. BE : Best Effort

7. QoS : Quality of Service

8. UL $L_{\text {subframe }}$ : UL subframe

9. $H_{D L}$ : Header of DL subframe

10. $H_{U L}$ : Header of UL subframe

11. L:constant $=U L_{-}$symbols $+D L_{-}$symbols

12. $D L_{\text {subframe }}:$ DL subframe

13. UL_symbols :number of symbols occupied in each UL subframe

14. DL_symbols :number of symbols occupied in each DL subframe

15. 15. $\bar{Q}_{U L}$ : average UL queue size during last $\mathrm{x}$ subframes

16. $16 . \bar{Q}_{D L}$ : average $\mathrm{DL}$ queue size during last $\mathrm{x}$ subframes

17. 17. $x$ :integer value

\section{Algorithm}

1. The incoming DL data and UL request traffic are considered by the BS.

2. The incoming traffic is then categorized into RT, NRT and $\mathrm{BE}$, based on its QoS features, by the BS.

3. The QoS requirement of the RT users are maximum delay tolerance and maximum allowable packet dropping rate.

4. The QoS requirement of the NRT users are minimum transmission rate.

5. The remaining users without any strict QoS requirement are considered as the BE users.

6. Thus, based on QoS features, all of the incoming traffic is classified into one of the three types.

7. Subframes of DL data traffic and UL request traffic are allocated dynamically to generate apppropriate DL/UL subframe ratio.

8. The UL subframe is allocated according to the equation (1).

$$
U L_{\text {subframe }}=\frac{L \alpha \beta-H_{D L} \beta+H_{U L} P \beta}{P \beta+\alpha}
$$

Where

$$
\alpha=\frac{D L_{\text {subframe }}}{D L_{-} \text {symbols }} \quad \beta=\frac{U L_{\text {subframe }}}{U L_{-} \text {symbols }} \quad P=\frac{\bar{Q}_{D L}}{\bar{Q}_{U L}}
$$

9. The DL subframe is allocated according to te equation (2).

$$
D L_{\text {subframe }}=\frac{L \alpha \beta-\alpha \cdot U L_{\text {subframe }}}{\beta}
$$

10. The DL/UL subframe ratio is calculated by dividing equation (1) by equation (2).

Thus, the DL/UL subframe ratio is dynamically estimated according to the DL data and UL request traffic.

\subsection{Prioritization of the Users}

After the dynamic computation of the DL/UL subframe ratio, the users are prioritized. Based on the priority, the users are processed. This process is described in algorithm 2 .

\section{Algorithm 2}

Notations:

1. $\mathrm{k}$ :user

2. $\mathrm{K}$ :total number of users

3. $Q_{k}$ :Priority level of user k

4. $q_{R T}$ :basic priority of RT user

5. $\quad D_{k}$ :QoS measure of delay experienced by the first packet of RT user

6. $D_{k}^{*} \quad$ :QoS requirement of the highest tolerable delay for the RT user

7. $q_{N R T}$ :basic priority of NRT user

8. $R_{k}^{*}$ :QoS requirement of the minimum transmission rate for NRT user k

9. $\quad R_{k}^{\prime}$ :QoS measure of the average transmission rate for user
10. $q_{B E}$ :basic priority of $\mathrm{BE}$ user

11. $Q_{k}^{*}$ :Priority Threshold for user k

12. $R_{k}$ :Allocated transmission rate

13. $A_{k}$ :number of bits required by $\mathrm{k}$ to keep QoS requirement guaranteed

14. CSI:Channel State Information

15. $\operatorname{CSI}_{k}^{(n)}$ :Channel State Information of sub channel $\mathrm{n}$ received at user $\mathrm{k}$

16. $n \quad$ sub channel

17. $\operatorname{SINR}_{k}^{(n)} \quad$ :Signal to Interference plus Noise Ratio on $\mathrm{n}$ received at $\mathrm{k}$

18. $\operatorname{SINR}_{k}^{*}(M) \quad$ SINR on $\mathrm{n}$ received at k by applying M QAM modulation order

19. $M$ : order of QAM modulation $=\{4,16,64\}$

\section{Algorithm:}

1. For $\mathrm{K}$ total users with $\mathrm{k}$ being one among the total users such that $0 \leq k \leq K$, the $Q_{k}$ is estimated for every user using equation (3).

$Q_{k}=q_{R T} * \exp \left(\frac{D_{k}+1}{D_{k}^{*}}\right) ;$ if $\mathrm{k}$ is a RT user

$\begin{array}{ll}=q_{N R T} * \exp \left(\frac{1.5 R_{k}^{*}-0.5 R_{k}^{\prime}}{R_{k}^{*}}\right) ; & \text { if } \mathrm{k} \text { is a NRT user } \\ =q_{B E} ; \quad \text { if } \mathrm{k} \text { is BE user }\end{array}$

2. Then the $Q_{k}$ value is compared with $Q_{k}^{*}$.

3. If $Q_{k}<Q_{k}^{*}$, then the user $\mathrm{k}$ is assigned lower priority.

4. If $Q_{k}>Q_{k}^{*}$, then the user $\mathrm{k}$ is assigned higher priority and are considered for processing immediately.

5. In this way, the priority of every user is determined.

6. Then the higher priority users are processed one by one in the decreasing order of its priority at a data amount of $D_{k}$ and at a transmission rate, $R_{k}$.

7. After all the higher priority users are processed, then the lower priority users are considered.

8. The CSI value of the lower priority users are estimated using equation (4).

$\operatorname{CSI}_{k}^{(n)}=1 ;$ if $\operatorname{SINR}_{k}^{*}(4) \leq \operatorname{SINR}_{k}^{(n)} \leq \operatorname{SINR}_{k}^{*}(16)$

$=2 ;$ if $\operatorname{SINR}_{k}^{*}(16) \leq \operatorname{SINR}_{k}^{(n)} \leq \operatorname{SINR}_{k}^{*}(64)$

$=3 ;$ if $\operatorname{SINR}_{k}^{*}(64) \leq \operatorname{SINR}_{k}^{(n)}$

$=0 ;$ otherwise

9. User $\mathrm{k}$ with the highest CSI value, $C S I_{k}^{(n)}$ on subchannel $\mathrm{n}$ is scheduled.

10. If two or more users on subchannel $n$ have same CSI value, then the user which has greater $Q_{k}$ value is selected and scheduled for the accessing the sub channel $n$.

11. In this way, scheduling of all the low priority users for accessing each sub channel is continued until the resources are exhausted or till there is no user in the queue which has any data to be transmitted.

Thus, all the users are prioritized into either higher priority user or lower priority user. Then based on the priority level, the users are processed.

\subsection{User Acceptance Based on the Power Consumption Rate}

Efficient data transmission is possible if the sub channel allocation and scheduling is performed appropriately. If a user allocated with a sub channel consumes higher transmission power, then this allocation must be changed since it is basically a lower priority user and hence can even be scheduled at a later point of time. This enables the utilization of the sub channels by users which consume lesser transmission power. Thus, in turn minimizing the power consumption rate and allowing effective usage of the sub 
channels. This process is described in algorithm 3 .

Algorithm 3:

Notations:

1. $\mathrm{N}$ :sub channel

2. $\mathrm{P}_{\mathrm{T}}^{\text {old(n) }}$ :Transmission power through $\mathrm{n}$ prior scheduling based on priority

3. $\mathrm{P}_{\mathrm{T}}^{\text {new }(\mathrm{n})}$ :Transmission power through $\mathrm{n}$ after scheduling based on priority

\section{Algorithm:}

1. Initially, the power consumed, for the transmission of the lower priority data through the available sub channel is determined.

2. After scheduling the sub channels for all the lower priority users, the total transmission power, for each user through the scheduled sub channel are estimated.

3. The value is compared with the value.

4. If , then the sub channel allocated to the user is finalized and data is processed through it.

5. If , then the associated user is removed and the sub channel is used for other users as per the network requirement.

In this way, the sub channels are utilized efficiently by appropriate users. Thus, the power consumption rate is minimized which in turn ensures effective network performance.

\section{Simulation Results}

\subsection{Simulation Parameters}

We utilize NS2 to mimic our Priority Based Dynamic Resource Allocation and Scheduling for WiMAX Networks (PBDRAS) convention. We utilize the IEEE 802.16 for WiMAX arranges as the MAC layer convention. It has the usefulness to inform the system layer about connection breakage. In our recreation, the rate is differed as $1,1.5,2,2.5$ and $3 \mathrm{Mb}$. The region estimate is 1000 meter $\mathrm{x} 1000$ meter square locale for 50 seconds reproduction time. The reenacted movement is Constant Bit Rate (CBR).

Our simulation settings and parameters are summarized in table 1

Table 1: Simulation parameters

\begin{tabular}{|l|l|}
\hline No. of Nodes & 12 \\
\hline Area & $1000 \mathrm{X} 1000$ \\
\hline MAC & 802.16 \\
\hline Simulation Time & $50 \mathrm{sec}$ \\
\hline Traffic Source & CBR \\
\hline Flows & 12 \\
\hline Propagation & TwoRayGround \\
\hline Antenna & OmniAntenna \\
\hline Rate & $1,1.5,2,2.5$ and $3 \mathrm{Mb}$ \\
\hline
\end{tabular}

\subsection{Performance Metrics}

We evaluate performance of the new protocol mainly according to the following parameters. We compare the QDRA [11] protocol with our proposed PBDRAS protocol.

Bandwidth: It is the number of mega bits transmitted to the receiver.

Fairness: It is the ratio between total received bandwidth and total available bandwidth.

Delay: It is the time taken to reach the destination by the data packets.

\subsection{Results \& Analysis}

The simulation results are presented in the next section.

Case-1(CBR Traffic)

In our first experiment we vary the transmission rate as $1,1.5,2,2.5$ and $3 \mathrm{Mb}$.

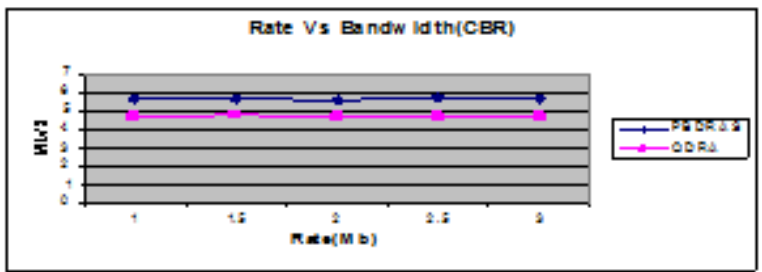

Fig 2: Rate Vs Bandwidth

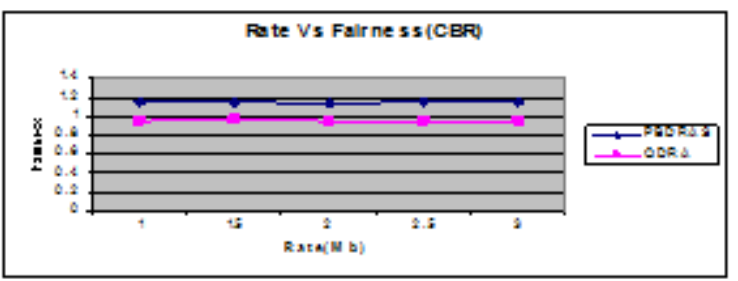

Fig 3: Rate Vs Fairness

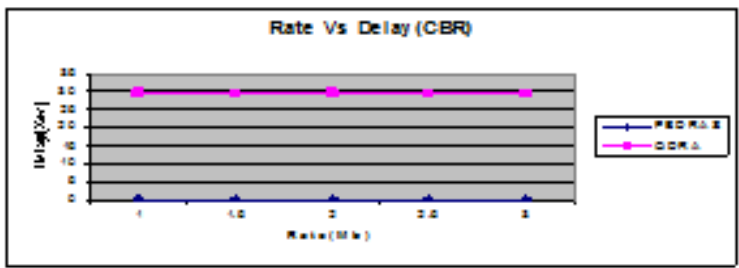

Fig 4: Rate Vs Delay

Figures 2 to 4 demonstrate the consequences of bandwidth,fairness and deferral by changing the rate from $1 \mathrm{Mb}$ to $3 \mathrm{Mb}$ for the CBR movement in PBDRAS and QDRA conventions. When looking at the execution of the two conventions, we construe that PBDRAS beats QDRA by $17 \%$ regarding data transfer capacity, $17 \%$ as far as fairnes and $99 \%$ as far as deferral.

Case-2 (EXP-Traffic)

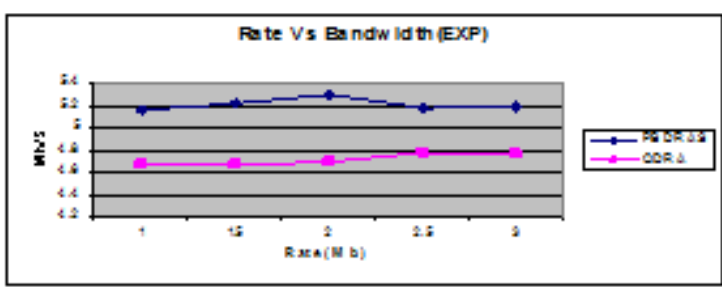

Fig 5: Rate Vs Bandwidth

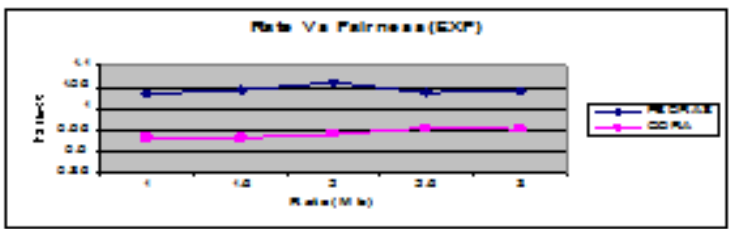

Fig 6: Rate Vs Fairness

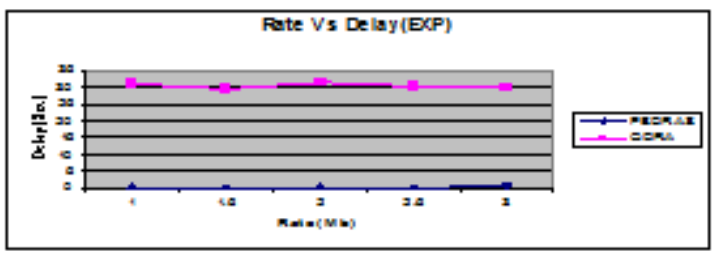

Fig 7: Rate Vs Delay

Figures 5 to 7 demonstrate the aftereffects of transmission capacity, reasonableness and postponement by shifting the rate from $1 \mathrm{Mb}$ to $3 \mathrm{Mb}$ for the CBR activity in PBDRAS and QDRA conventions. When contrasting the execution of the two conventions, we induce that PBDRAS beats QDRA by $9 \%$ as far as transfer speed, 9\% regarding decency and $99 \%$ as far as 
postponement.

\section{Case-3(Video-Traffic)}

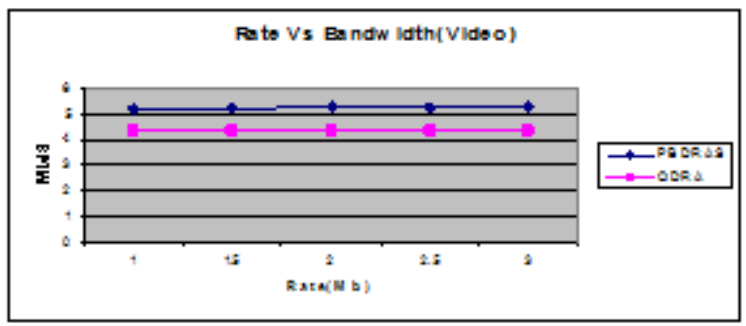

Fig 8: Rate Vs Bandwidth

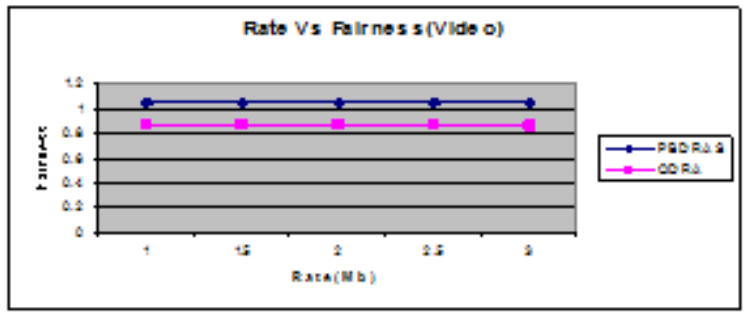

Fig 9: Rate Vs Fairness

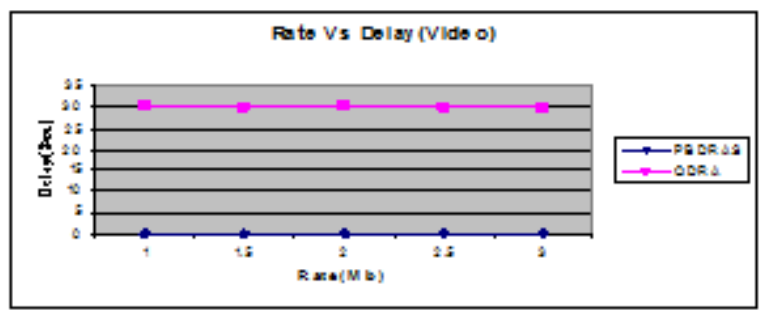

Fig 10: Rate Vs Delay

Figures 8 to 10 demonstrate the aftereffects of transmission capacity, reasonableness and postponement by differing the rate from $1 \mathrm{Mb}$ to $3 \mathrm{Mb}$ for the CBR activity in PBDRAS and QDRA conventions. When contrasting the execution of the two conventions, we deduce that PBDRAS beats QDRA by $17 \%$ regarding transfer speed, $17 \%$ as far as fairnes and $99 \%$ as far as postponement.

\section{Conclusion}

In this paper, we have proposed Priority Based Dynamic Resource Allocation and Scheduling for WiMAX Networks. In this allocation and scheduling technique, initially the incoming traffic sub frames are dynamically allocated to avoid overloading the channel during data transmission. The incoming traffic is also categorized into real time, non real time and best effort data. Next, the users are prioritized and the higher priority data are transmitted instantly. But, the low priority data are scheduled and allocated to corresponding sub channels with respect to the CSI value. Finally, the power consumed in data transmission through sub channels with and without prioritized scheduling is compared. If the scheduling performed w.r.t CSI value does not reduce the power consumption, then the user is removed from the allocation. In this way, the critical data are served with utmost priority and also network power consumption is maintained minimal, thus ensuring effective network performance.

\section{References}

[1] T.L. Economopoulos, P.A. Asvestas, G.K. Matsopoulos, “Contrast enhancement of images using Partitioned Iterated Function Systems", Image and Vision Computing Volume 28, Issue 1, January 2010

[2] Fadi Abu-Amara and Ikhlas Abdel-Qader, "HybridMammogram
Classification Using Rough Set and Fuzzy Classifier", International Journal of Biomedical Imaging, Volume 2009, July 2009.

[3] B. Surendiran and A. Vadivel, " A hybrid classifier for mammogram mass classification using various new geometric shape and margin features”, Int. J. Rapid Manufacturing, Vol. 2, Nos. 1/2, pp.56-75, 2011.

[4] Amal AlQoud and M. Arfan Jaffar, "Hybrid Gabor based Local Binary Patterns Texture Features for classification of Breast Mammograms", International Journal of Computer Science and Network Security, VOL.16 No. 2, February 2016.

[5] M. Arfan Jaffar, "Hybrid Texture based Classification of Breast Mammograms using Adaboost Classifier", International Journal of Advanced Computer Science and Applications, Vol. 8, No. 5, 2017.

[6] R.Nithya, B.Santhi, "Mammogram Classification Using Maximum Difference Feature Selection Method", Journal of Theoretical and Applied Information Technology, Vol. 33 No.2, November 2011.

[7] Ping Zhang, Kuldeep Kumar, and Brijesh Verma, "A Hybrid Classifier for Mass Classification with Different Kinds of Features in Mammography”, FSKD, LNAI 3614, pp. 316 - 319, 2005.

[8] Ankita Satyendra Singh, M. M. Pawar, "Mass Classification of Mammogram Images using Selected Textural Features with PNN Classifier" International Journal of Innovative Research in Computer and Communication Engineering, Vol.5, Special Issue 4, June 2017.

[9] Jinchang Ren, DongWang, JianminJiang, "Effective recognition of MCCs in mammograms using an improved neural classifier" Engineering Applications of Artificial Intelligence 24,638 $645,2011$.

[10] R. Swiniarski Hun Ki Lim, "Independent Component Analysis, Principal Component Analysis and Rough Sets in Hybrid Mammogram Classification", International SPIE Symposium Medical Imaging, 2003.

[11] S Murali and P Sathees Kumar, "Multiclass classification of mammogram images with GLCM features", International Journal of Engineering Research Science and Technology, Vol. 4, No. 1, February 2015 